\title{
Erratum to: Estimating reliability and resolution of probability forecasts through decomposition of the empirical score
}

\author{
Jochen Bröcker
}

Published online: 12 October 2012

(c) Springer-Verlag Berlin Heidelberg 2012

\section{Erratum to: Clim Dyn (2012) 39:655-667 DOI 10.1007/s00382-011-1191-1}

Unfortunately, the paper "Estimating reliability and resolution of probability forecasts through decomposition of the empirical score," Climate Dynamics, 2012, contains a few errors. Corrections for these errors are provided here.

\section{The ignorance score}

In the relations (20-22), $\rho_{d}$ and $r_{d}$ have to swap places. The correct reading of these relations is

$$
\begin{aligned}
& \mathrm{e}(\bar{\pi}) \cong \mathrm{e}(\bar{o})+\frac{K-1}{2 N} \\
& \sum_{d=1}^{D} \mathrm{~d}\left(\bar{\pi}, \pi^{(d)}\right) \rho_{d} \cong \sum_{d=1}^{D} \mathrm{~d}\left(\bar{o}, o^{(d)}\right) r_{d}-\frac{(K-1)(D-1)}{2 N}, \\
& \sum_{d=1}^{D} \mathrm{~d}\left(\gamma^{(d)}, \pi^{(d)}\right) \rho_{d} \cong \sum_{d=1}^{D} \mathrm{~d}\left(\gamma^{(d)}, o^{(d)}\right) r_{d}-\frac{(K-1) D}{2 N}
\end{aligned}
$$

\section{The Brier score}

Below Eqs. (23-25), the definition of $v_{d}$ should read $v_{d}=1-\left(1-\rho_{d}\right)^{N}$, as in the appendix.

The online version of the original article can be found under doi:10.1007/s00382-011-1191-1.

J. Bröcker $(\bowtie)$

Department of Mathematics and Statistics, University of

Reading, PO BOX 220, Reading RG6 6AX, UK

e-mail: j.broecker@reading.ac.uk
Immediately after that, it is claimed that the term in brackets on the right-hand side of Eq. (24) is never negative, which would imply that the empirical resolution overestimates the true resolution. For the Brier score, this statement is wrong in general, and the proof (unnumbered equation just after Eq. 25) is faulty. It is assumed that the entropy $e$ is convex, while in fact it is concave. However, it is worth noting that the term in question is positive for many choices of $\pi^{(d)}, \rho_{d}, d=1 \cdots D$. To make this more precise, note that for the Brier score, $\mathrm{e}(p)=1-\sum_{k} p_{k}^{2}$. Therefore, the relation

$\sum_{d} \mathrm{e}\left(\pi^{(d)}\right) v_{d} \geq \mathbf{e}(\bar{\pi})$

evidently holds under the condition that the $\pi^{(d)}$ live in a sphere of radius $\sqrt{1-\frac{\mathrm{e}(\bar{\pi})}{\sum_{d} v_{d}}}$, centered at the origin. A simple calculation shows that $\mathrm{e}(\bar{\pi}) \leq \frac{K-1}{K}$, while the $v_{d}$ are very nearly equal to one, unless $N$ is very small, as discussed. We can conclude that the empirical resolution overestimates the true resolution if the $\pi^{(d)}$ are restricted to a sphere of radius no more than $\sqrt{1-\frac{K-1}{K D}}$.

It is maybe worth mentioning that although the empirical decomposition does not necessarily overestimate the resolution term, it does overestimate the potential score (see Eq. 30); statements in the paper to that effect are correct.

Finally, a minor error occured in the appendix: The plus symbol in the unnumbered, displayed equation immediately preceding Eq. (32) should be a minus symbol.

Acknowledgments The fact that the bias in the resolution term is not necessarily positively oriented was pointed out to me by Christopher Ferro, who also helped with spotting a few additional errors. 